EPJ Web of Conferences 116, 11003 (2016)

DOI: $10.1051 /$ epjconf/201611611003

(C) Owned by the authors, published by EDP Sciences, 2016

\title{
KM3NeT-ARCA project status and plan
}

\author{
R. Coniglione ${ }^{\mathrm{a}}$ on behalf of the KM3NeT Collaboration \\ INFN, Laboratori Nazionali del Sud, via S. Sofia 62, Catania 95123, Italy
}

\begin{abstract}
The KM3NeT Collaboration aims at building a research infrastructure in the depths of the Mediterranean Sea hosting a cubic kilometre neutrino telescope. The KM3NeT/ARCA detector is the ideal instrument to look for high-energy neutrino sources thanks to the latitude of the detector and to the optical characteristics of the sea water. The detector latitude allows for a wide coverage of the observable sky including the region of the Galactic centre and the optical sea water properties allow for the measure of the neutrino direction with excellent angular resolution also for cascade events. The technologically innovative components of the detector and the status of construction will be presented as well as the capability it offers to discover neutrinos.
\end{abstract}

\section{Introduction}

The main aims of the KM3NeT Collaboration are the observation of cosmic high energy neutrinos and the study of their origins and the determination of the neutrino mass hierarchy. To reach these goals the Collaboration started the construction of a network of two telescopes in the depth of the Mediterranean Sea: ARCA, with an instrumented volume of about $1 \mathrm{~km}^{3}$ that will be installed near the Sicilian (Italy) coast, and ORCA, a smaller and more densely instrumented detector for the study of the neutrino mass hierarchy that will be installed near the Toulon coast (France). In this paper the status and performances of the KM3NeT/ARCA detector will be described while the KM3NeT/ORCA detector is discussed in [1]. The detection principle of the under sea high-energy neutrino detectors is based on the measurement of the Cherenkov light induced in water by the secondary charged particles produced in neutrino interactions inside or in the vicinity of the detector.

The study of high energy neutrinos produced in galactic and extragalactic sources can shed light on the production mechanisms of high energy particles and can give important constraints on the acceleration models being neutrinos a strong signature of the presence of hadronic processes. The IceCube observation of the high-energy astrophysical neutrino flux [2,3] has marked a turning point in astro-particle physics. The presence of neutrino of cosmic origin states that high energy particles are produced in the Cosmos with a neutrino flux of intensity detectable by the present generation of high energy neutrino telescopes. The sources producing high energy neutrinos have not been yet determined

\footnotetext{
a e-mail: coniglione@lns.infn.it
}

This is an Open Access article distributed under the terms of the Creative Commons Attribution License 4.0, which permits unrestricted use, distribution, and reproduction in any medium, provided the original work is properly cited. 
and the spectral shape and flavour composition have not been precisely defined [4]. Also the claimed isotropic distribution of the measured neutrino flux [3] requires further experimental confirmation.

KM3NeT/ARCA is a complementary instrument to IceCube: located in the Northern hemisphere and exploiting sea water as medium for the Cherenkov light propagation it has a different field of view and presents different systematics uncertainties. The good angular resolution, both for cascade and track events, and the large visibility of the Galactic Plane makes ARCA the ideal instrument to investigate Galactic sources and in general all sources located in the Southern sky. This unique characteristics has pushed the collaboration to optimize the detector design for the detection of neutrinos from Galactic sources which have an expected energy from a few $\mathrm{TeV}$ to a few $10 \mathrm{TeV}$. The confirmation of the diffuse neutrino flux measured by IceCube is expected as one of the first physics goal of the Collaboration.

\section{The KM3NeT/ARCA detector design}

The arrival times of the light collected by optical sensors and their geometrical positions are used to reconstruct the tracks of the particles produced in neutrino interactions and from that also the neutrino direction and its energy. Large detectors with a size of a cubic-kilometer arranged in a 3-dimensional array of photo sensors are required.

One of the concept that has been validated in the past years by the KM3NeT Collaboration is that the overall sensitivity is not reduced if the neutrino telescope is split into separate detector building blocks [5]. The consequent detector modularity complies with the technical specifications for the construction and maintenance of the infrastructure. KM3NeT/ARCA will be constituted by two building blocks, each one of 115 Detection Units (DU) having an instrumented volume of about $0.5 \mathrm{~km}^{3}$. The DUs are flexible structures anchored at the sea floor, kept vertical by buoys and connected to shore by an electro-optical cable network. Each DU hosts 18 Digital Optical Modules (DOM). Each DOM consists of a pressure resistant glass sphere that contains 313 inches photomultipliers and the readout electronics. The horizontal distance between strings is about $90 \mathrm{~m}$ and the vertical distance between adjacent optical modules is $36 \mathrm{~m}$.

A brief description of two technologically innovative elements of the detector, the DU and the DOM, is given hereafter. A description of the other parts of the detector as the trigger and data acquisition system, the time calibration, the positioning system, the electronics, the data transmission system, the live monitoring and the GRID computing system is available in the other KM3NeT contributions to this workshop.

The DOM [6,7] is a pressure-resistant glass sphere of 17-inch diameter hosting 31 3-inch photomultiplier tubes (PMTs) with low power high-voltage bases and read-out electronics. Each tube is surrounded by a reflector that effectively increases the collection efficiency per PMT by about $27 \%$. The lower hemisphere of each DOM contains 19 of the PMTs, downward-looking, whereas the other 12 PMTs in the upper hemisphere are up-looking. The optical contact between the PMTs and the glass sphere is assured by optical gel. The front-end electronics [8] amplify the PMT signals and transform them into digital time-over-threshold information that is fed into the readout via optical fibres. All PMT signals above an adjustable threshold (typically the equivalent of 0.3 photo-electrons) are sent to shore, where event candidates are selected by online filters running on a computer farm. The DOM contains three calibration sensors: a LED nano-beacon for time calibration, a compass and tiltmeter for orientation calibration and an acoustic piezo sensor, glued to the inner surface, for position calibration. A DOM mounted on a DU is shown in Fig. 1 left.

This innovative photon sensor, which is the result of an intense R\&D activity, has several advantages with respect to the conventional optical modules with one 10 inch PMT. Its photocathode area is 3 to 4 times the area of a single PMT module and has an almost uniform angular coverage. The photocathode segmentation allows for a more efficient identification of more than one photon arriving at the DOM. This improves the background rejection and provides directional information. These features have 

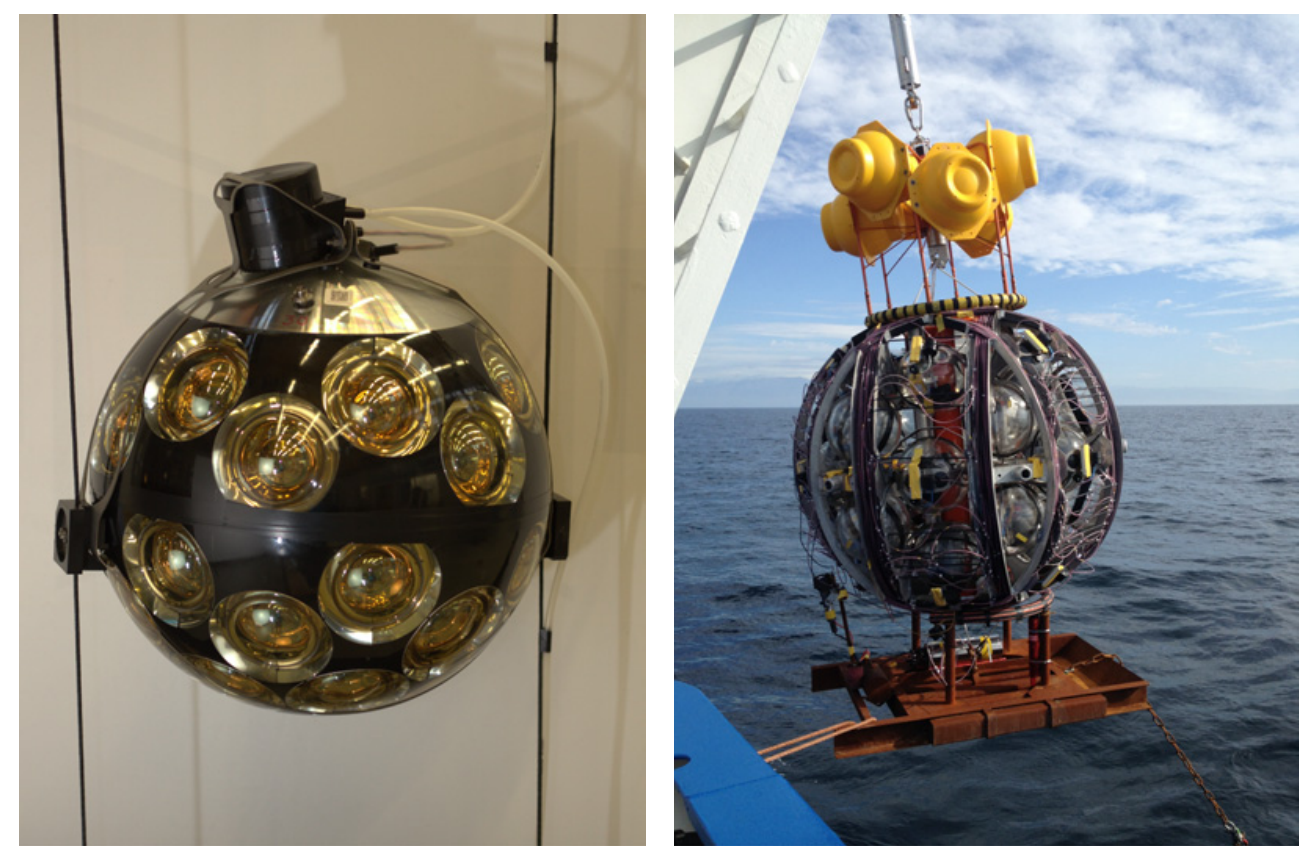

Figure 1. Left - The DOM fixed on the two parallel Dyneema ${ }^{\circledR}$ ropes. Right - The deployment of the LOM vehicle.

been confirmed by the analysis of data collected with a DOM prototype [9] hosted on the ANTARES instrumented line [10] and with the DU prototype [11] deployed at the Italian site and operated for 9 months. A procedure for time calibration which exploits ${ }^{40} \mathrm{~K}$ decays and the LED beacons inside the DOM has been developed for the DU prototype and tested, reaching a precision at the level of nanosecond.

The DU [12] is a slender structure consisting of two parallel Dyneema ${ }^{\circledR}$ ropes to which the DOMs are attached via a titanium collar (see Fig.1 left). Attached to the ropes is the vertical electro-optical cable, a pressure balanced, oil-filled, plastic tube that contains two copper wires and 18 optical fibres for the power and data transmission. At each DOM two power conductors and a single fibre are branched out via the breakout box. The breakout is the black box mounted on the top of the glass sphere in Fig. 1 left.

For the deployment, a detection unit is wound around a spherical frame with diameter of about $2.2 \mathrm{~m}$ (Launcher of Optical Modules, LOM [13]), which is posed on the seabed and after an acoustic release command starts unwinding the DU with a rotating upwards movement. At the end of operation the LOM reaches the sea surface, where it is collected for reuse. A picture of the LOM ready for the deployment is shown in Fig. 1, right.

\section{The status of the KM3NeT/ARCA detector}

The KM3NeT/ARCA detector will consist of 2 building blocks, each with 115 DUs. The design of the detector allows for a staged construction. Funding for the first phase of the KM3NeT (Phase-1) is already available. It foresees the deployment of:

- 24 string-like DUs and 8 DUs based on a flexible tower design [14] at the Italian site.

- 7 string-like DUs at the French site with a denser distribution of the optical sensors, representing the first nucleus of the ORCA detector [1]. 


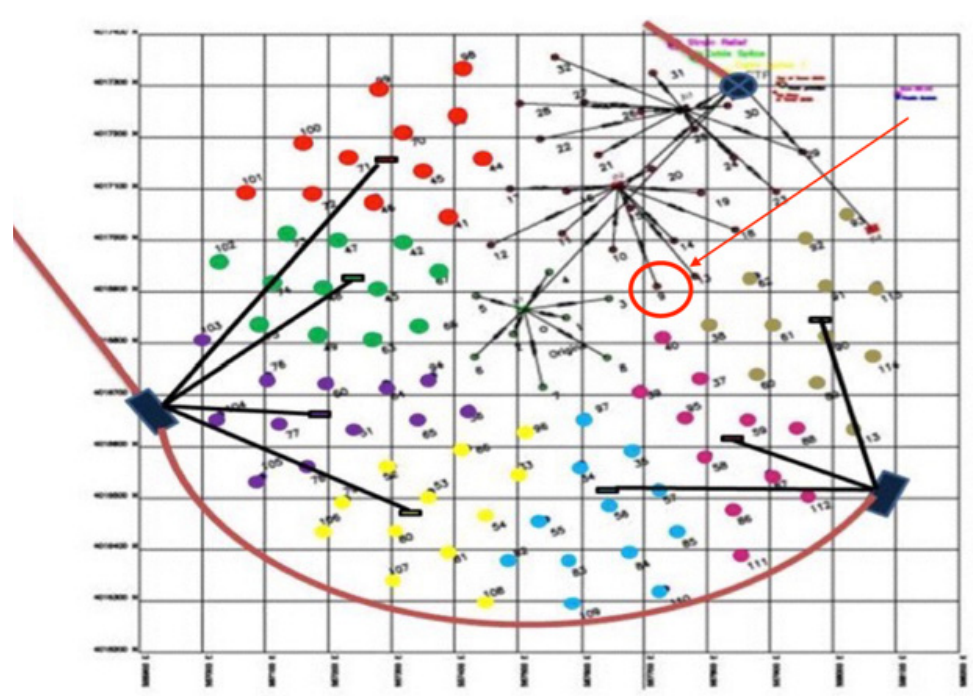

Figure 2. Footprint of one ARCA building block. The position of the first DU is indicated with the red arrow.

The volume of the ARCA Phase- 1 detector is about $0.1 \mathrm{~km}^{3}$ and, when completed, it will be the largest neutrino telescope in the Northern hemisphere.

DOMs and DUs integration sites have been already established. Two DUs have been already integrated and fully tested. One of them has been successfully deployed on December 3rd 2015 at the Italian site. The deployment, at $3500 \mathrm{~m}$ under the sea level smoothly validated the entire procedure and the functionality of the sea floor infrastructure. Data taking and analysis started a few hours after switching on the apparatus. In Fig. 2 the layout of the ARCA detector is shown and the position of the first DU indicated with a red arrow.

\section{Physics performances}

Two classes of events can be identified in a high energy neutrino telescope: track-like events and cascade-like events. The track-like events are generated mainly by muons that are produced in the matter inside or surrounding the detector through $v_{\mu} \mathrm{CC}$ interactions. The cascade-like events are produced in the matter near or inside the detector volume mainly through $v_{e} \mathrm{CC}$ interactions and NC interactions of neutrinos of all flavours. These two categories of events produce very different time-space hit patterns in the detector. Dedicated reconstruction algorithms exploiting the characteristic hit patterns have been developed in order to get the direction and the energy of the neutrinos. The good optical properties of the sea-water and the directional response of the DOM make possible to achieve angular resolutions of about $0.2^{\circ}$ for track events for $v_{\mu}$ above $10 \mathrm{TeV}$ and $2^{\circ}$ for cascade events with $v_{e}$ energy above $50 \mathrm{TeV}$. A very promising track reconstruction algorithm, that include detailed knowledge of the detector, is in its final developing stage and the first tests show that the angular resolution can be improved to a level of $0.1^{\circ}$.

The primary strategy for the search of neutrino sources is the identification of up-going muons produced by neutrinos crossing the Earth. Thanks to the location in the Northern hemisphere, the field of view of the KM3NeT detector includes a large part of the Galactic plane. In this region of the sky the most promising neutrino sources are expected [15]. In addition to its favourable position, the good angular resolution makes the ARCA detector the ideal tool to search for neutrino sources. Its sensitivity 

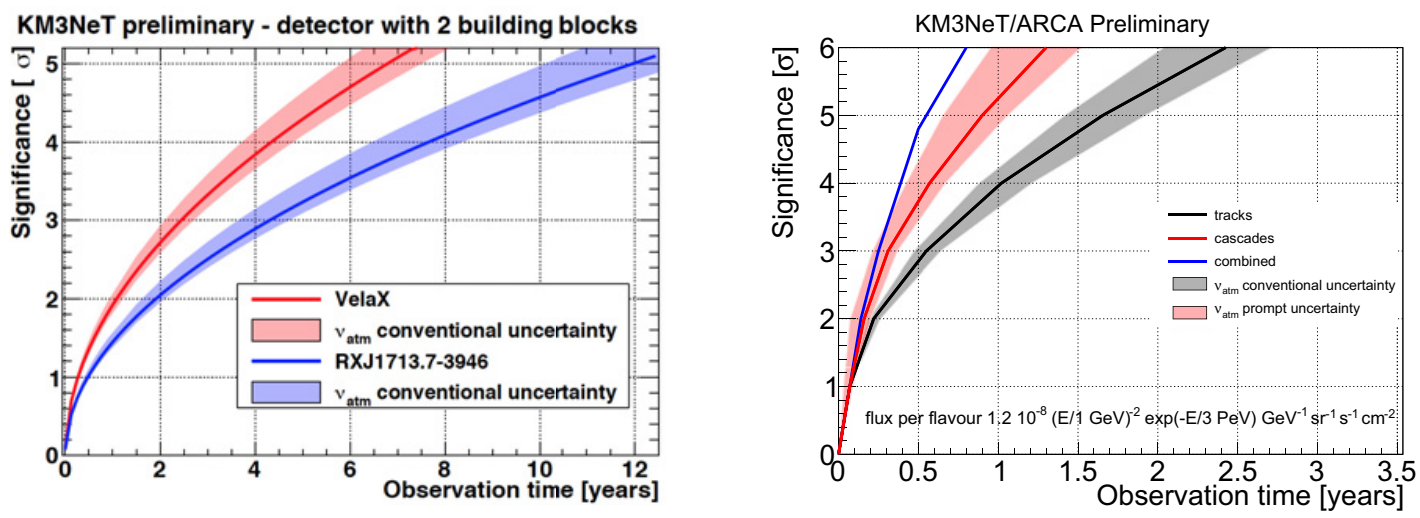

Figure 3. Significance as a function of the observation time for the detection of: Left - the Galactic sources RXJ1713 and Vela-X. The bands represent the uncertainties due to the conventional component of the atmospheric neutrino flux (see Ref. [16]); Right - a diffuse flux of neutrinos corresponding to the signal reported by IceCube for the cascade channel (red line) and muon channel (black line). The black and red bands represent the uncertainties due to the conventional and prompt component of the neutrino atmospheric flux (see Ref. [18]). The blue line represents the results of the combined analysis.

to Galactic point-like source has been estimated by means of Monte Carlo simulations [16] looking for up-going muon neutrino tracks. Some Galactic sources that are the most intense high energy gamma ray sources and possible neutrino emitters, the Super Nova Remnants RXJ1713 and Vela Junior and the Pulsar Wind Nebula VelaX have been studied in particular. The RXJ1713 and the Vela X neutrino spectrum have been estimated considering the hypothesis of transparent sources and a fully hadronic production mechanism [16, 17]. The measured extension of the source in $\gamma$-rays has been taken into account in the simulation. Results from simulations show that a significance of about 3 sigma is reached in 3 years (Fig. 3 left).

The confirmation of the measurement of the diffuse flux by IceCube, with different methodology, improved resolution and complementary field of view, is an important goal for KM3NeT. Moreover, the better angular resolution to cascade events can constraint the region of the sky from where these neutrinos origin. The sensitivity of the ARCA detector to the neutrino flux measured by IceCube has been evaluated using both track-like events reconstructed up to $10^{\circ}$ above the horizon and cascade-like events in the full angular range [18]. The results of this analysis are summarized in Fig. 3 right. A significance of 5 sigma can be reached in less than one year.

\section{Summary}

In this contribution the KM3NeT/ARCA project has been briefly presented, the main technical components of the detector and performance have been described. The construction of the detector, which in its final configuration consists of 2 building blocks of 115 DUs with a total instrumented volume of about $1 \mathrm{~km}^{3}$, has begun. KM3NeT-Phase1 is fully funded and will consist of 24 strings and 8 towers, at the Italian site near Capo Passero (Sicily), and 7 strings at the French site, close to the Toulon coast.

In December 2015 the first DU of the ARCA detector has been deployed and the first data files have been analyzed. All the DOMs are functional.

The detector sensitivities to the neutrino fluxes from Galactic sources and to the diffuse flux measured by IceCube have been discussed for the full ARCA detector. 


\section{References}

[1] D. Samtleben (KM3NeT collaboration), in this workshop (2015)

[2] M.G. Aartsen et al. (IceCube collaboration), Science 342, 1242856 (2013)

[3] M.G. Aartsen et al. (IceCube collaboration), Astrophys. J. 809, 98 (2015)

[4] M.G. Aartsen et al. (IceCube collaboration), PRL 114, 171102 (2015)

[5] M. de Jong (KM3NeT collaboration), Proc. 33 ${ }^{\text {rd }}$ Int. Cosmic Ray Conference 1, 0891 (2013)

[6] R. Bruijn, D. van Eijk (KM3NeT collaboration), Proc. $34^{\text {th }}$ Int. Cosmic Ray Conference 001, 1157 (2015)

[7] D. Vivolo (KM3NeT collaboration), in this workshop (2015)

[8] D. Real (KM3NeT collaboration), in this workshop (2015)

[9] S. Adrián-Martínez et al. (KM3NeT collaboration), Eur. Phys. J. C 74, 3056 (2014)

[10] M. Ageron et al., NIM A 656(1), 11 (2011), ISSN 0168-9002, http://www. sciencedirect. com/science/article/pii/S0168900211013994

[11] S. Adrián-Martínez et al. (KM3NeT collaboration) (2015), arXiv : 1510.01561 [astro-ph. IM]

[12] P. Kooijman (KM3NeT collaboration), Proc. $34^{\text {th }}$ Int. Cosmic Ray Conference 001, 1173 (2015)

[13] E. de Wolf et al. (KM3NeT collaboration), NIM A 725, 241 (2013)

[14] S. Aiello et al., Astroparticle Physics 66, 1 (2015), ISSN 0927-6505, http://www.science direct.com/science/article/pii/S0927650514001960

[15] A. Kappes, J. Hinton, C. Stegmann, F. Aharonian, Astrophys. J. 656, 870 (2007), erratum in Astrophys. J. 661 (2007) 1348, arXiv: astro-ph/0607286

[16] A. Trovato et al. (KM3NeT collaboration), in this workshop (2015)

[17] S. Kelner, F. Aharonian, V. Bugayov, Phys. Rev. D 74, 034018 (2006), erratum in Phys. Rev. D 78039901 (2009), arXiv: 0802.0562 [physics.ins-det]

[18] L. Fusco et al. (KM3NeT collaboration), in this workshop (2015) 